\title{
Long-term evolution of the neutron-star spin period of SXP $1062 \star$
}

\author{
R. Sturm ${ }^{1}$, F. Haberl ${ }^{1}$, L. M. Oskinova ${ }^{2}$, M. P. E. Schurch ${ }^{3}$, V. Hénault-Brunet ${ }^{4}$, J. S. Gallagher III $^{5}$, and A. Udalski ${ }^{6}$ \\ ${ }^{1}$ Max-Planck-Institut für extraterrestrische Physik, Giessenbachstraße, 85748 Garching, Germany \\ e-mail: rsturm@mpe.mpg.de \\ 2 Institute for Physics and Astronomy, University Potsdam, 14476 Potsdam, Germany \\ 3 Astrophysics, Cosmology and Gravity Centre (ACGC), Astronomy Department, University of Cape Town, Rondebosch, \\ Private Bag X1 7701, South Africa \\ ${ }^{4}$ Scottish Universities Physics Alliance (SUPA), Institute for Astronomy, University of Edinburgh, Blackford Hill, \\ Edinburgh EH9 3HJ, UK \\ 5 Department of Astronomy, University of Wisconsin-Madison, 5534 Sterling, 475 North Charter Street, Madison, WI 53706, USA \\ ${ }^{6}$ Warsaw University Observatory, Aleje Ujazdowskie 4, 00-478 Warsaw, Poland
}

Received 22 April 2013 / Accepted 2 July 2013

\section{ABSTRACT}

\begin{abstract}
Context. The Be/X-ray binary SXP 1062 is of especial interest owing to the large spin period of the neutron star, its large spin-down rate, and the association with a supernova remnant constraining its age. This makes the source an important probe for accretion physics.

Aims. To investigate the long-term evolution of the spin period and associated spectral variations, we performed an XMM-Newton target-of-opportunity observation of SXP 1062 during X-ray outburst.

Methods. Spectral and timing analysis of the XMM-Newton data was compared with previous studies, as well as complementary Swift/XRT monitoring and optical spectroscopy with the SALT telescope were obtained.

Results. The spin period was measured to be $P_{\mathrm{s}}=(1071.01 \pm 0.16) \mathrm{s}$ on 2012 Oct. 14 . The X-ray spectrum is similar to that of previous observations. No convincing cyclotron absorption features, which could be indicative for a high magnetic field strength, are found. The high-resolution RGS spectra indicate the presence of emission lines, which may not completely be accounted for by the SNR emission. The comparison of multi-epoch optical spectra suggest an increasing size or density of the decretion disc around the Be star.
\end{abstract}

Conclusions. SXP 1062 showed a net spin-down with an average of $\dot{P}_{\mathrm{s}}=(2.27 \pm 0.44) \mathrm{s} \mathrm{yr}^{-1}$ over a baseline of 915 days.

Key words. pulsars: individual: SXP1062 - galaxies: individual: Small Magellanic Cloud - stars: emission-line, Be - stars: neutron X-rays: binaries

\section{Introduction}

Be/X-ray binaries (BeXRBs, Reig 2011) are the dominant subclass of high-mass X-ray binaries in the Small Magellanic Cloud (SMC) with more than 100 known systems (including candidates). These systems comprise of a neutron star (NS) that accretes matter from the circumstellar decretion disc of an emission-line star of spectral class B (Be star) leading to, often transient, X-ray emission.

The BeXRB binary SXP 1062 was discovered by Hénault-Brunet et al. (2012) using Chandra and XMM-Newton observations of the star-forming region NGC 602 (Oskinova et al. 2013b). Amongst other BeXRBs in the SMC, SXP 1062 is outstanding owing to: (i) The position in the wing of the SMC, where a younger stellar population is found and less BeXRBs are known compared to the bar of the SMC. (ii) A relatively long spin period of the NS of $P_{\mathrm{s}} \sim 1062 \mathrm{~s}$, making it

* Based on observations obtained with XMM-Newton, an ESA science mission with instruments and contributions directly funded by ESA Member States and NASA and on observations made with the Southern African Large Telescope (SALT).

$\star \star$ The reduced SALT spectra is only available at the CDS via anonymous ftp to cdsarc.u-strasbg. fr $(130.79 .128 .5)$ or via http://cdsarc.u-strasbg.fr/viz-bin/qcat?]/A+A/556/A139 the second slowest known pulsar of the SMC (after SXP 1323, Haberl \& Pietsch 2005). (iii) A rapid spin-down of the NS of $\dot{P}_{\mathrm{s}}=0.26 \mathrm{~s} \mathrm{~d}^{-1}$ (Haberl et al. 2012) that was observed over the interval between 2010 Mar. 25 and Apr. 12 (18 days), whereas in general spin-up is observed during enhanced accretion. (iv) A robust correlation with a supernova remnant (SNR) was found by Hénault-Brunet et al. (2012). Owing to the low density of both BeXRBs and SNRs in the wing of the SMC, this correlation is unlikely by chance. The SNR is clearly seen in optical emission lines ( $\mathrm{H} \alpha$ and [O III]). Additional radio emission and a detailed analysis of the X-ray emission of the SNR can be found in Haberl et al. (2012). The SNR constrains the age of the NS to 10000-25000 years, making SXP 1062 a relatively young BeXRB.

These peculiar properties have made SXP 1062 the subject of several theoretical investigations, with the main issue of explaining the long spin period of this young NS. Suggested scenarios include an initially slow spin period (Haberl et al. 2012), an initially and/or presently strong magnetic field (Popov \& Turolla 2012; Fu \& Li 2012), and the accretion of magnetised matter (Ikhsanov 2012).

Monitoring of Galactic accreting pulsars revealed that individual NSs can switch between spin-up and spin-down phases 
over short time scales down to a few days and that the NS spins up during intervals of high accretion and spins down over X-ray quiescent periods (Bildsten et al. 1997). This raises the question if the large spin-down of SXP 1062 was observed by chance and if the source continued to spin down with such a high $\dot{P}_{\mathrm{s}}$.

An outburst of SXP 1062 in 2012 was found in the optical with OGLE and in X-rays with the Swift satellite. In this paper, we present our analysis of an XMM-Newton target-ofopportunity observation of SXP 1062 in Oct. 2012, which allows to measure the long-term evolution of the spin period of the NS on a longer time scale of $\sim 900$ days since the first XMM-Newton measurement. All uncertainties in this paper are given for $1 \sigma$ confidence.

\section{Observations and data reduction}

On 2012 Oct. 9, SXP 1062 was found in X-ray outburst with the Swift satellite. The evolution of the X-ray flux was followed in a monitoring campaign since then (TargetID: 32580). The XRT spectra were reduced from the cleaned level-3 event files within a circle placed on the source with the ftool ${ }^{1}$ xselect. Background spectra were created from a nearby point-sourcefree circular extraction region. The ancillary response files were calculated with xrtmkarf.

The detection of SXP 1062 in X-ray bright state allowed us to ask for an XMM-Newton (Jansen et al. 2001) follow-up observation (ObsID: 0700381801) that was performed on 2012 Oct. 14. SXP 1062 was observed on-axis with all EPIC instruments in full-frame mode using the thin filter for EPIC-pn (Strüder et al. 2001) and the medium filter for both EPICMOS (Turner et al. 2001). We processed the data using the XMM-Newton SAS 12.0.12. Because the flaring background was at moderate level (below 8 and 3 counts s$^{-1} \operatorname{arcmin}^{-2}$ for pn and MOS, respectively), no temporal screening of the data was necessary. This yields an exposure of 26.6, 31.1, and $31.2 \mathrm{ks}$ for EPIC-pn, -MOS1, and -MOS2, respectively. Energy spectra and time series of the source and the background were extracted, using the same selection regions as described in Haberl et al. (2012, see their Fig. 1, region A and B) such that emission of the SNR will be subtracted. We used single- and doublepixel events from EPIC-pn and single- to quadruple-pixel events from EPIC-MOS. For energy spectra we rejected events with FLAG $\neq 0$. This resulted in 24810,8009 , and 8908 net counts in the $(0.2-12.0) \mathrm{keV}$ band for the three instruments. We binned the EPIC spectra to have a signal-to-noise ratio of $\geq 5$ for each bin. Spectra from the Reflection Grating Spectrometer (RGS, den Herder et al. 2001) with an exposure of $31.6 \mathrm{ks}$ were extracted with rgsproc. We used events within a cross-dispersion point-spread-function width of $90 \%$, which excludes roughly half of the area covered by the SNR. The background events were selected for a point-spread-function width $>99 \%$, to exclude emission from the NS and the SNR. The resulting spectra contain 277 (RGS1) and 380 (RGS2) net counts in the (0.35-2.0) keV band. The RGS spectra were binned with 25 net cts $\mathrm{bin}^{-1}$. Although these spectra have low statistics, they provide the first grating X-ray data of SXP 1062.

We used the Southern African Large Telescope (SALT, Buckley et al. 2006a,b; O’Donoghue et al. 2006 to collect long

\footnotetext{
1 http://heasarc.nasa.gov/ftools/

2 Science Analysis Software (SAS), http://xmm.esac.esa.int/ sas/
}

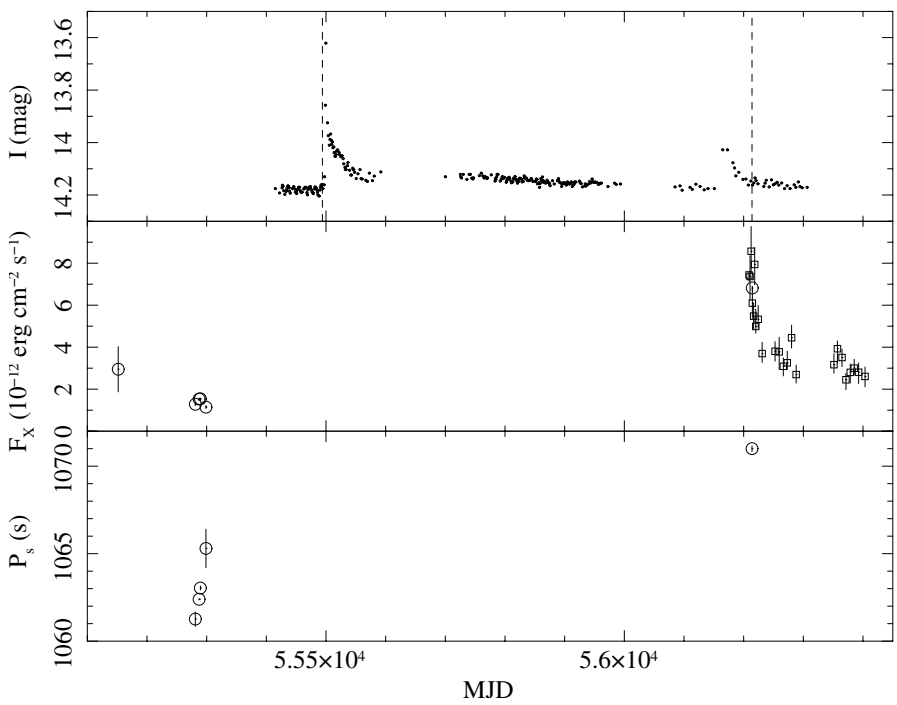

Fig. 1. Upper panel: OGLE-IV I-band light curve. Dashed lines indicate the time of optical spectroscopy observations. Middle panel: $\mathrm{X}$-ray fluxes in the $(0.2-10.0) \mathrm{keV}$ band from Swift (open squares) and XMM-Newton (open circles) including the 2009 slew-survey data and the 2010 measurements. Lower panel: NS spin period as measured with XMM-Newton.

slit spectra of SXP 1062 simultaneous with the XMM-Newton observation. As part of the SALT program 2012-1-RSA_UKSC003 (PI: Schurch) aimed at studying outbursts from X-ray binary systems, we obtained spectra of SXP 1062 during the evening of 2012 Oct. 13th and morning of the 14th. These data were collected using the Robert Stobie Spectrograph (RSS, Burgh et al. 2003; Kobulnicky et al. 2003). We used the PG2300 grating in two position angles $\left(30.500^{\circ}\right.$, and $\left.48.875^{\circ}\right)$ to achieve coverage from (3835-4924) $\AA$ in the blue and (6082-6925) $\AA$ in the red, respectively. To achieve the highest resolution we used a $0.6^{\prime \prime}$ wide slit. Observations were prebinned by a factor 2 producing a spectral resolution of $0.33 \AA$ per binned pixel. Two 400 s observations were taken in the blue on MJD 56213.99 followed by a $180 \mathrm{~s}$ exposure of a ThAr arc lamp. A single $180 \mathrm{~s}$ exposure was taken in the red on MJD 56214.01 followed by a $90 \mathrm{~s}$ exposure of an Ar arc lamp. All observations were flat fielded using quartz tungsten halogen (QTH) flats taken immediately after the blue and before the red observations. The image quality during the observations was approaching $3^{\prime \prime}$. An hour after the observations a spectrophotometric standard star (LTT1020) was observed for both the blue and red setups. The SALT data was first processed using the SALT pipeline, PySALT (Crawford et al. 2010). This performs overscan, gain, cross-talk corrections, and mosaicing of the three $2048 \times 4096$ CCDs. Subsequent flatfielding, background subtraction, wavelength calibration and extraction of 1D spectra were performed in IRAF v2.16 (Tody 1993). The final spectra were first redshift corrected by $150 \mathrm{~km} \mathrm{~s}^{-1}$ and normalised (after being averaged in the case of the two blue $400 \mathrm{~s}$ exposures).

An optical monitoring of SXP 1062 is provided by the Optical Gravitational Lensing Experiment (OGLE, Udalski et al. 2008) that covers the source regularly with I-band photometry since the beginning of phase IV (2010 Aug. 6). The data are available in the X-Ray variables OGLE Monitoring (XROM, Udalski 2008) system and presented in the upper panel of Fig. 1. 
Table 1. Spectral fit results.

\begin{tabular}{lccccccccc}
\hline \hline Model $^{a}$ & $\begin{array}{c}N_{\mathrm{H}, \mathrm{smc}} \\
{\left[10^{21} \mathrm{~cm}^{-2}\right]}\end{array}$ & $\Gamma$ & $\begin{array}{c}k T \\
{[\mathrm{eV}]}\end{array}$ & $N^{b}$ & $\begin{array}{c}E W_{\mathrm{Fe}} \\
{[\mathrm{eV}]}\end{array}$ & $\begin{array}{c}F^{c} \\
{\left[\mathrm{erg} \mathrm{cm}^{-2} \mathrm{~s}^{-1}\right]}\end{array}$ & $\begin{array}{c}L^{d} \\
{\left[\mathrm{erg} \mathrm{s}^{-1}\right]}\end{array}$ & $\begin{array}{c}\chi_{v}^{2} \\
\text { d.o.f. }\end{array}$ \\
\hline PL & $1.54 \pm 0.09$ & $0.774 \pm 0.009$ & - & - & - & $(6.8 \pm 0.7) \times 10^{-12}$ & $3.0 \times 10^{36}$ & 1.01 & 1199 \\
PL+BB & $1.99 \pm 0.18$ & $0.723 \pm 0.014$ & $237 \pm 22$ & $11.2_{-2.1}^{+2.7} \mathrm{~km}$ & - & $(6.9 \pm 0.2) \times 10^{-12}$ & $3.1 \times 10^{36}$ & 0.99 & 1197 \\
PL+DiskBB & $2.32 \pm 0.24$ & $0.728 \pm 0.014$ & $308 \pm 42$ & $6.2_{-1.7}^{+1.4} \mathrm{~km}$ & - & $(6.9 \pm 0.2) \times 10^{-12}$ & $3.1 \times 10^{36}$ & 0.99 & 1197 \\
PL+DiskBB+Fe & $2.30 \pm 0.25$ & $0.733 \pm 0.015$ & $307 \pm 41$ & $6.0_{-1.6}^{+2.3} \mathrm{~km}$ & $40 \pm 13$ & $(6.9 \pm 0.2) \times 10^{-12}$ & $3.1 \times 10^{36}$ & 0.98 & 1196 \\
PL+APEC & $1.78 \pm 0.11$ & $0.729 \pm 0.011$ & $1059 \pm 113$ & $5.1_{-0.9}^{+1.2} \times 10^{57} \mathrm{~cm}^{-3}$ & - & $(6.9 \pm 1.0) \times 10^{-12}$ & $3.1 \times 10^{36}$ & 0.98 & 1197 \\
\hline
\end{tabular}

Notes. All uncertainties are given for $\Delta \chi^{2}=1$ corresponding to a $1 \sigma$ confidence for one degree of freedom. ${ }^{(a)}$ For model definition see text. (b) Normalisation of the thermal model component: radius (BB), inner disc radius for inclination of $\Theta=0$ (DiskBB), and emission measure (APEC). ${ }^{(c)}$ Observed flux in the $(0.2-10.0) \mathrm{keV}$ band. ${ }^{(d)}$ Unabsorbed luminosity in the $(0.2-10.0) \mathrm{keV}$ band. A source distance of $60 \mathrm{kpc}$ is assumed.

\section{Analyses and results}

\subsection{X-ray energy spectrum}

Spectral analysis was performed with xspec (Arnaud 1996) version 12.7.0u. We used the same model, as described in Haberl et al. (2012), i.e. an absorbed power law where we assume Galactic photoelectric absorption with a column density of $N_{\mathrm{H} \text {,gal }}=6 \times 10^{20} \mathrm{~cm}^{-2}$ with solar abundances according to Wilms et al. (2000), and a free column density, $N_{\mathrm{H}, \mathrm{smc}}$, with abundances set to 0.2 solar accounting for absorption by the interstellar medium of the SMC or within the BeXRB system. The normalisations between the individual instruments were found to be consistent, and we fitted the same model to all five spectra simultaneously. The spectrum and best-fit model are presented in Fig. 2a and the best-fit parameters are listed in Table 1. Formally, the power-law model describes the data well with a reduced $\chi_{v}^{2}$ of 1.01. However, in other BeXRBs, a soft excess (Hickox et al. 2004) and iron fluorescent emission are believed to contribute to the X-ray spectrum.

A possible $\mathrm{Fe} \mathrm{K}_{\alpha}$ emission line broadened below the instrumental resolution was investigated by fitting an additional Gaussian emission-line profile with central energy of $E_{\mathrm{c}}=$ $6.4 \mathrm{keV}$, line width of $\sigma=0$ and free normalisation. We obtain a $3.1 \sigma$ evidence for a line with normalisation of $N=(3.1 \pm 1.0) \times$ $10^{-6}$ photons $\mathrm{cm}^{-2} \mathrm{~s}^{-1}$, corresponding to an equivalent width of $E W=(-40 \pm 13) \mathrm{eV}$. Analogously, we derive for ionised Fe XXV $\left(E_{\mathrm{c}}=6.7 \mathrm{keV}\right)$ a $3 \sigma$ upper limit of $N<2.9 \times 10^{-6}$ photons $\mathrm{cm}^{-2} \mathrm{~s}^{-1}$ and $N<3.1 \times 10^{-6}$ photons $\mathrm{cm}^{-2} \mathrm{~s}^{-1}$ for Fe XXVI $\left(E_{\mathrm{c}}=7.0 \mathrm{keV}\right)$.

To investigate a possible soft excess and to demonstrate systematic uncertainties of the power-law and absorption parameters we fitted additional thermal components. Adding a blackbody $(\mathrm{BB})$ emission component to the model results in a slight improvement of the fit with an f-test probability of $2.3 \times 10^{-7}$. This formally proves the significance of this component, however see Protassov et al. (2002) for limitations of the f-test. We further fitted a multi-temperature accretion disc model (DiskBB) and a collisionally ionised plasma model with 0.2 solar abundances (APEC). The results are presented in Table 1. The possible contribution of the disc black-body model and $\mathrm{Fe} \mathrm{K}_{\alpha}$ line are demonstrated in Fig. 2b, where we omit the RGS spectra for clarity. The residuals of this model, rebinned by a factor of 5, are compared with the residuals of the simple power-law model in Figs. $2 d$ and c. We find that e.g. in the case of a disc black-body a soft excess can only contribute with a luminosity of $7.5_{-1.1}^{+0.8} \times 10^{34} \mathrm{erg} \mathrm{s}^{-1}$, i.e. $2.5_{-0.4}^{+0.3} \%$ of the unabsorbed luminosity in the $(0.2-10.0) \mathrm{keV}$ band.

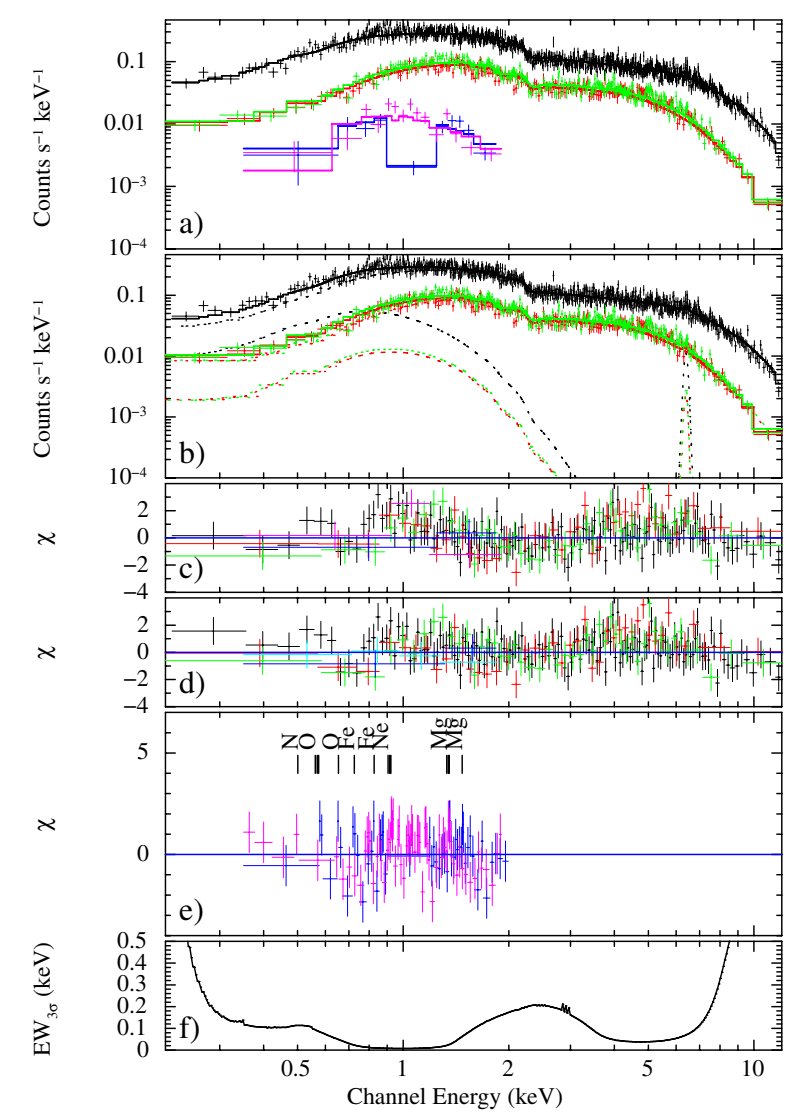

Fig. 2. a) Energy spectrum of SXP 1062 as observed on 2012 Oct. 14 with EPIC-pn (black), -MOS1 (red), -MOS2 (green), RGS1 (blue), and RGS2 (magenta) together with the best-fit model (solid line) of an absorbed power law. b) Same as a), but fitted with additional contribution of a multi-temperature disc black-body model and $\mathrm{Fe} \mathrm{K}_{\alpha}$ emission line, shown by dashed lines. The RGS data are omitted for clarity. c) The residuals in units of $\sigma$ for the model from a) with a higher binning by a factor of 5. d) Same as c), but for the model from b). e) The residuals for the model of a), binned with only 5 net cts bin ${ }^{-1}$. Vertical lines mark the energies of prominent emission lines. f) $3 \sigma$ upper limits for the equivalent width (EW) of a Gaussian absorption line.

To check for spectral features in the RGS grating spectra, we plot the residuals of the power-law fit with a binning of only 5 net cts bin $^{-1}$. We find clear indications of emission lines of the helium-like triplets of O VII $(0.561-0.574 \mathrm{keV})$, Ne IX (0.905-0.922 keV), and Mg XI (1.33-1.35 keV) as well as hydrogen-like N VII $(0.500 \mathrm{keV})$, O VIII $(0.654 \mathrm{keV})$, and 




Fig. 3. Power-density spectrum of SXP1062. The best-fit frequency $\omega=934 \mu \mathrm{Hz}$ and harmonics are marked by vertical lines.

Mg XII (1.47 keV) and neon-like Fe XVII (0.725, $0.826 \mathrm{keV})$, as marked in Fig. 2e. Due to the low statistics in the spectra, we can only roughly constrain the fluxes of the most convincing lines to $1.6_{-0.8}^{+0.9} \times 10^{-5}$ photons $\mathrm{cm}^{-2} \mathrm{~s}^{-1}$ (O VII), $4.9_{-2.5}^{+3.4} \times 10^{-6}$ photons $\mathrm{cm}^{-2} \mathrm{~s}^{-1}$ (O VIII), and $1.3_{-0.4}^{+0.5} \times 10^{-5}$ photons $\mathrm{cm}^{-2} \mathrm{~s}^{-1}$ (Ne IX). Here we used the unbinned data and C statistics (Cash 1979).

The peculiar properties of SXP 1062 were suggested to be caused by a strong magnetic field ( $\left.B \sim 10^{14} \mathrm{G}, \mathrm{Fu} \& \mathrm{Li} 2012\right)$, possibly leading to a proton cyclotron feature in the observed energy band. Adding a Gaussian absorption line to the powerlaw model, we find no significant contribution of this feature. Stepping the absorption line through the complete spectrum, we estimate $3 \sigma$ upper limits for EW as shown in Fig. 2f, where we assume a line width of $\sigma=0.2 E_{\mathrm{c}}$.

\subsection{X-ray pulsations}

A fast Fourier transformation of the merged EPIC time series (42 $231 \mathrm{cts}$ including background) reveals the pulsation of the NS and various harmonics as presented in Fig. 3. Using a Bayesian detection method as described by Gregory \& Loredo (1996) and Haberl et al. (2008), the most probable spin period during the recent XMM-Newton observation is derived to $P_{\mathrm{s}}=$ $(1071.01 \pm 0.16) \mathrm{s}$.

The background-subtracted normalised folded light curves are shown in Fig. 4 for the total $(0.2-10.0) \mathrm{keV}$ band and the standard sub-bands (0.2-0.5) keV, (0.5-1.0) keV, (1.0-2.0) keV, (2.0-4.5) keV, and (4.5-10.0) $\mathrm{keV}$, where we merged the first two bands to increase the statistics. The HRs are defined by $\mathrm{HR}_{i}=\left(\mathrm{R}_{i+1}-\mathrm{R}_{i}\right) /\left(\mathrm{R}_{i+1}+\mathrm{R}_{i}\right)$ with $\mathrm{R}_{i}$ denoting the backgroundsubtracted count rate in the XMM-Newton standard energy band $i$ (with $i$ from 1 to 4 ). In Fig. 4, we also show the pulse profile measured in 2010 (ObsID 0602520201), folded with 1062.4 s. The phase offsets are set to have a common maximum in the total-band light curves.

\subsection{Long-term X-ray light curve}

The position of SXP 1062 was covered with the Einstein satellite, but no source is reported in the catalogue of Wang \& Wu (1992). Also the ASCA catalogue of Yokogawa et al. (2003)
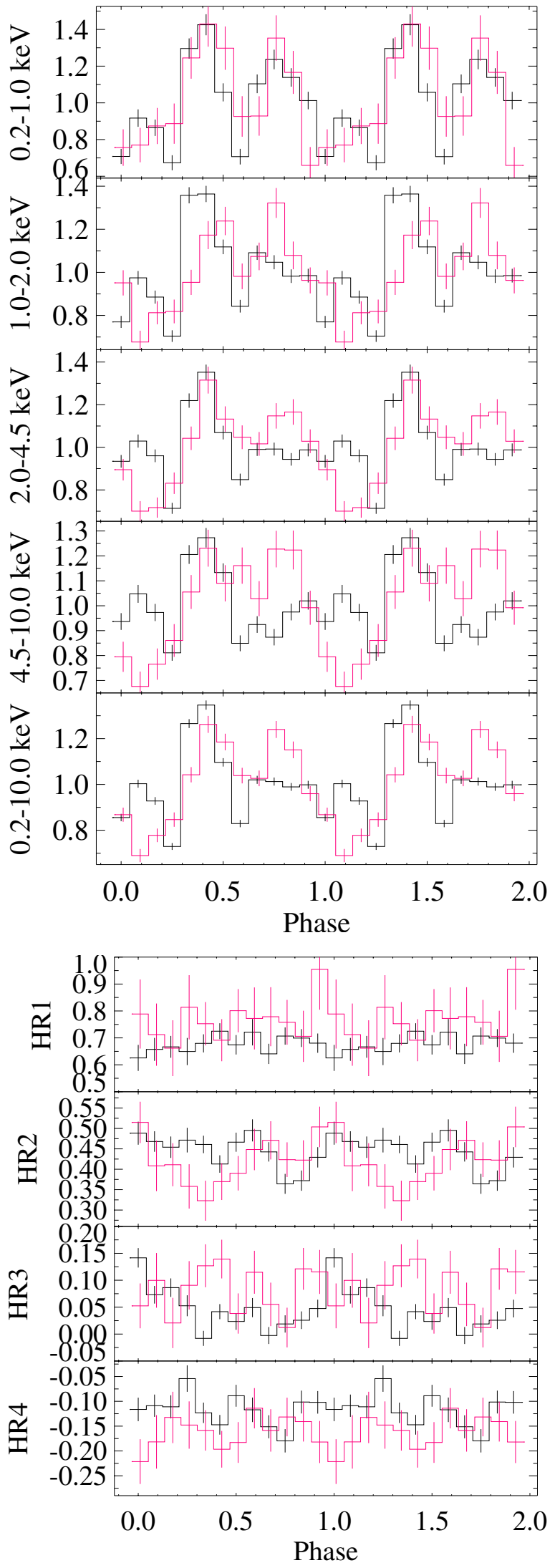

Fig. 4. Upper figure: pulse profile of the time series, merged from all EPIC instruments in different energy bands for the 2012 observation (black) and an observation in 2010 (red). The individual light curves are background subtracted and normalised to the average count rate (from top to bottom: $0.19,0.40,0.44,0.35,1.37 \mathrm{cts} \mathrm{s}^{-1}$ in 2012 and 0.020 , $0.044,0.052,0.037,0.15 \mathrm{cts} \mathrm{s}^{-1}$ in 2010). Lower figure: hardness ratios as function of pulse phase. 


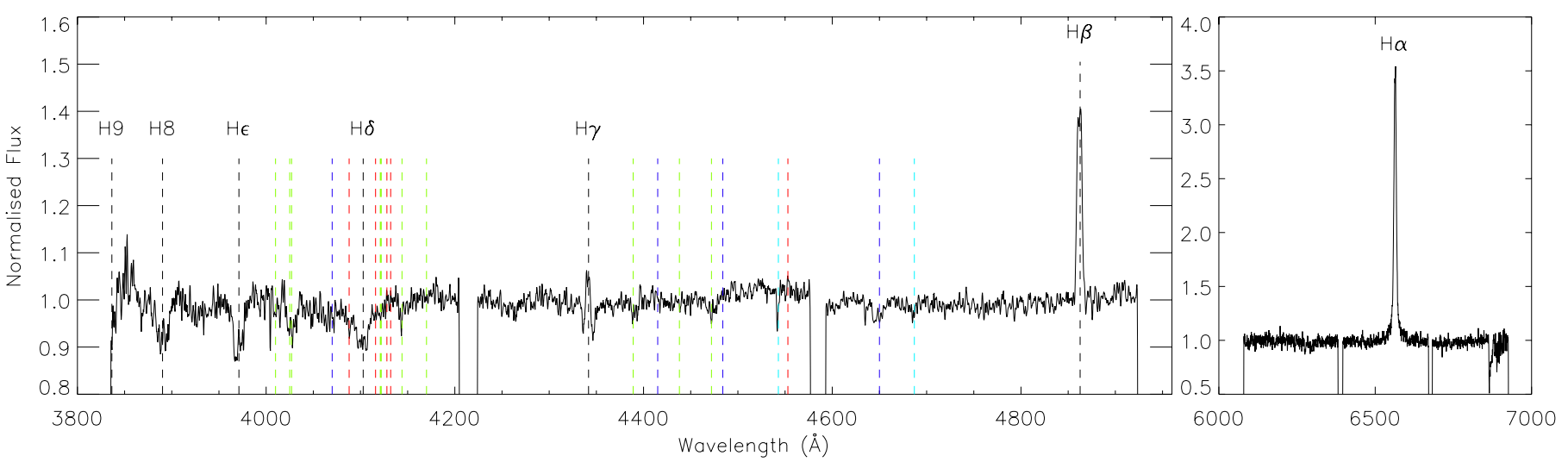

Fig. 5. SALT blue and red (left and right respectively) smoothed spectra of SXP 1062. Clearly visible are the chip gaps between the three CCDs. Dotted lines indicate: Balmer lines (black), He I (green), He II (light blue), Silicon (red) and other metal lines (dark blue).

does not contain SXP 1062, which was in the FoV of an observation performed on 1999 May 28 to 29.

We note that both catalogues list a nearby source (No. 67 of Wang \& Wu 1992 and No. 105 of Yokogawa et al. 2003). The angular separation of $\sim 4.4^{\prime}$ is too large for a correlation with SXP 1062 with respect to the angular resolution of both satellites. In the recent XMM-Newton observation, as well as in the 2010 observations, we do not find a bright source at this position. Hence, the nearby source might be an X-ray transient. In the case of another BeXRB, the closest possible counterparts are 2dFS 3857 (B0-5V star, 60" angular separation), and 2MASS J01281201-7330235 (B3-5III star showing near-infrared variability, 80").

Three pointed ROSAT observations of SMC X-1 covered the position of SXP 1062 between 1991 and 1993 (MJD 48 536.2, 48 895.7, and 49141.0). For these observations, no detection at the position of SXP 1062 is reported in the catalogue of Haberl et al. (2000). Assuming 10 counts as a typical detection limit and the same spectral shape as measured in the recent XMM-Newton observation, we derive upper limits of 1.4, 2.7, and $2.1 \times 10^{-13} \mathrm{erg} \mathrm{cm}^{-2} \mathrm{~s}^{-1}$, respectively, which are the lowest limits for the X-ray flux reported so far.

As noted by Hénault-Brunet et al. (2012), the very first $\mathrm{X}$-ray detection of SXP 1062 is listed in the XMM-Newton slewsurvey catalogue (Saxton et al. 2008, Release 1.5). The source (XMMSL1 J012746.2-733304) was detected on 2009 Nov. 16 with $(4.2 \pm 1.5) \mathrm{cts}$, which according to the spectrum from above, results in a flux of $(3.0 \pm 1.1) \times 10^{-12} \mathrm{erg} \mathrm{cm}^{-2} \mathrm{~s}^{-1}$.

The recent Swift/XRT monitoring allows to follow the evolution of the X-ray outburst in 2012. Since the X-ray spectrum is measured well with XMM-Newton and we find no strong variations of the spectral shape compared to the 2010 observations, we fixed the spectral shape to the PL+DiskBB+Fe model and calculated fluxes for each Swift spectrum, using C statistics. The derived fluxes are listed in Table 2 . The X-ray light curve is presented in Fig. 1 and compared to the optical light curve from OGLE and the evolution of the spin period of the NS.

\subsection{Optical spectroscopy}

Figure 5 shows the fully reduced spectra after being smoothed using a boxcar average of 3 data points. The $\mathrm{H} \alpha$ and $\mathrm{H} \beta$ line are shown in more detail in Fig. 6. Neither line could be fitted well using one Gaussian alone. For $\mathrm{H} \alpha$, broad wings are seen in addition. $\mathrm{H} \beta$ clearly exhibits a double-peaked line profile that is also seen in the pre-subtraction images. Fitting both lines with two
Table 2. Swift/XRT observations of SXP 1062.

\begin{tabular}{|c|c|c|c|}
\hline $\mathrm{MJD}^{a}$ & $\begin{array}{l}\text { Net Exp } \\
{[\mathrm{s}]}\end{array}$ & $\begin{array}{c}\text { Rate } \\
{\left[10^{-2} \mathrm{~s}^{-1}\right]}\end{array}$ & $\begin{array}{c}\text { Flux }^{b} \\
{\left[10^{-12} \mathrm{erg} \mathrm{cm}^{-2} \mathrm{~s}^{-1}\right]}\end{array}$ \\
\hline 56209.01 & 15558 & $6.49 \pm 0.20$ & $7.44_{-0.31}^{+0.17}$ \\
\hline 56210.75 & 976 & $6.25 \pm 0.80$ & $7.37_{-1.13}^{+0.99}$ \\
\hline 56212.48 & 1019 & $7.36 \pm 0.85$ & $8.57_{-1.06}^{+1.18}$ \\
\hline 56214.62 & 1151 & $5.30 \pm 0.68$ & $6.10_{-0.72}^{+0.66}$ \\
\hline 56216.69 & 2078 & $4.72 \pm 0.48$ & $5.48_{-0.60}^{+0.62}$ \\
\hline 56218.56 & 1269 & $6.46 \pm 0.71$ & $7.94_{-0.97}^{+0.81}$ \\
\hline 56220.37 & 1748 & $4.06 \pm 0.48$ & $4.99_{-0.32}^{+0.82}$ \\
\hline 56224.44 & 1823 & $4.44 \pm 0.49$ & $5.33_{-0.42}^{+0.66}$ \\
\hline 56231.03 & 1723 & $2.96 \pm 0.41$ & $3.70_{-0.43}^{+0.54}$ \\
\hline 56252.70 & 1776 & $3.32 \pm 0.43$ & $3.81_{-0.47}^{+0.46}$ \\
\hline 56259.58 & 729 & $3.15 \pm 0.66$ & $3.78_{-0.85}^{+0.70}$ \\
\hline 56266.45 & 1895 & $2.22 \pm 0.34$ & $3.09_{-0.45}^{+0.41}$ \\
\hline 56273.06 & 2000 & $2.70 \pm 0.37$ & $3.26_{-0.33}^{+0.55}$ \\
\hline 56280.33 & 2188 & $3.93 \pm 0.42$ & $4.45_{-0.49}^{+0.60}$ \\
\hline 56288.01 & 1686 & $2.14 \pm 0.36$ & $2.70_{-0.41}^{+0.45}$ \\
\hline 56351.36 & 1940 & $2.83 \pm 0.38$ & $3.17_{-0.41}^{+0.52}$ \\
\hline 56357.17 & 1438 & $3.41 \pm 0.49$ & $3.93_{-0.69}^{+0.37}$ \\
\hline 56364.90 & 1878 & $3.14 \pm 0.41$ & $3.51_{-0.43}^{+0.40}$ \\
\hline 56371.51 & 2123 & $2.17 \pm 0.32$ & $2.45_{-0.47}^{+0.31}$ \\
\hline 56378.71 & 1950 & $2.36 \pm 0.35$ & $2.80_{-0.51}^{+0.41}$ \\
\hline 56385.40 & 1945 & $2.78 \pm 0.38$ & $3.00_{-0.32}^{+0.42}$ \\
\hline 56392.73 & 1201 & $2.66 \pm 0.47$ & $2.81_{-0.54}^{+0.44}$ \\
\hline 56403.51 & 1456 & $2.40 \pm 0.41$ & $2.61_{-0.50}^{+0.50}$ \\
\hline
\end{tabular}

Notes. ${ }^{(a)}$ Modified Julian date of the beginning of the observation. (b) Flux in the $(0.2-10.0) \mathrm{keV}$ band.

Gaussians each and using the unsmoothed data, we derive equivalent widths of the $\mathrm{H} \alpha$ and $\mathrm{H} \beta$ of $E W_{\mathrm{H} \alpha}=(-26.65 \pm 0.09) \AA$ and $E W_{\mathrm{H} \beta}=(-2.40 \pm 0.29) \AA$, respectively. Re-analysing previous $2 \mathrm{dF}$ spectra (Evans et al. 2004; Hénault-Brunet et al. 2012) with the same method, results in $E W_{\mathrm{H} \alpha}=(-22.02 \pm 0.054) \AA$ and $E W_{\mathrm{H} \beta}=(-1.58 \pm 0.10) \AA$. 

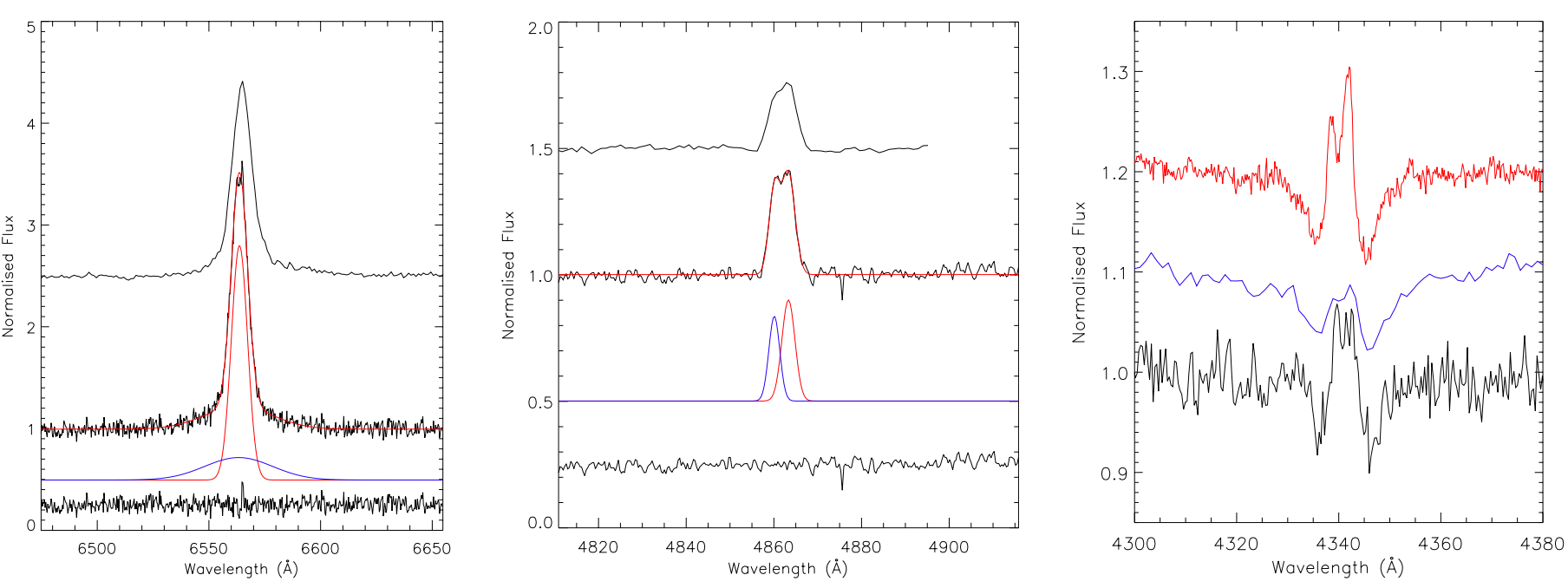

Fig. 6. Left: $\mathrm{H} \alpha$ as seen with SALT (normalised) and according modelling with two Gaussians and continuum. The lowest line gives the residuals. The $2 \mathrm{dF}$ spectrum is shown on the top for comparison. Middle: same as before, but for $\mathrm{H} \beta$. Right: $\mathrm{H} \gamma$ as seen with VLT FLAMES (top), 2dF (middle) and SALT (bottom).

\section{Discussion}

We analysed an XMM-Newton observation of SXP 1062 and find that the spin period of the NS further increased to $1071 \mathrm{~s}$. Since the last XMM-Newton observation, performed on 2010 Apr. 12, 915 days before the most recent observation, we obtain an average $\dot{P}_{\mathrm{s}}=(2.27 \pm 0.44) \mathrm{s} \mathrm{yr}^{-1}$. This is factor of 40 less than observed during the 18 day baseline in 2010. By assuming, that this average $\dot{P}_{\mathrm{s}}$ is representative for the NS since birth over $25 \mathrm{kyr}$, one derives an initial $P_{s}$ of $\sim 20 \mathrm{~s}$. If one assumes that the NS is born with a $P_{\mathrm{s}}$ of a few ms, a high initial magnetic field strength of $\sim 10^{14} \mathrm{G}$ (compared to a few $10^{12} \mathrm{G}$ as typically found for BeXRBs, e.g. Pottschmidt et al. 2012) and an efficient propeller mechanism are needed (e.g. Fu \& Li 2012) to slow the pulsar down within a few kyr to be close to the equilibrium period now.

The more moderate long-term spin-down rate suggests that the source was observed during high spin down phase in 2010 by chance and that the high spin-down rate is not an intrinsic feature of this source. This supports the hypothesis that SXP 1062 is rotating close to its equilibrium period, with spin-up during periods of high accretion rates and spin-down at other times. In this case, we would expect to observe spin-up of the NS occasionally as well, however there is no observational evidence so far. The recent data do not allow to conclude on the detailed evolution as periods of spin-up and -down may have alternated as seen in other BeXRBs. Especially the current $\dot{P}_{\mathrm{s}}$ cannot be determined from our single observation and the NS might even have shown spin-up during the X-ray outburst monitored by Swift. A denser $\mathrm{X}$-ray coverage of the source is highly desirable to determine the evolution of $P_{\mathrm{s}}$ and $\dot{P}_{\mathrm{s}}$ on a longer time scale and to constrain individual models.

Comparing the pulse profiles of 2010 and 2012, we find indications for a double peaked light curve in 2010, whereas in 2012 the light curve reveals only one clear peak at energies above $1 \mathrm{keV}$.

The shape of the X-ray spectrum is close to the 2010 measurement, but might contain a soft excess and an Fe K line. If the soft excess is caused by black-body emission, the black-body radius agrees with the radius of an NS and might originate from the NS surface. But also reprocessing of hard X-rays from the NS in nearby material like an accretion disc is possible, as we can only measure a lower limit of the inner disc radius, depending on the inclination $\left(R \sim \cos ^{-1 / 2} \Theta\right)$. Estimating the inner disc radius according to Hickox et al. (2004) yields $R=\left(L_{\mathrm{X}} / 4 \pi \sigma T^{4}\right)^{1 / 2}=$ $8.5 \mathrm{~km}$.

The high-resolution X-ray spectra from the RGS show indications of emission lines. The emission lines might be explained by the SNR around SXP 1062. However, assuming a homogeneous surface brightness of the SNR and typical SMC abundances of $Z=0.2 Z_{\odot}$, the best-fit plasma model for the SNR of Haberl et al. (2012) accounts only for a few percent of the observed O VII line flux. This points to an additional hot plasma component around the NS as it was e.g. observed in SXP 1323 (Haberl \& Pietsch 2005). Deeper X-ray observations of the source in an X-ray bright state are needed here, but we note that the line energies of the helium-like lines point to resonance lines rather than forbidden lines and that the emission of the observed He-like species is stronger than H-like like.

Proton cyclotron absorption lines (e.g. Zane et al. 2001) can be significant features in the X-ray spectra of NSs with high magnetic fields as observed with present-day observatories and might appear in the X-ray spectrum of SXP 1062 (Fu \& Li 2012). Neither in the residuals of the simple power-law fit (Fig. 2c) nor by adding an absorption-line component to the model (Sect. 3.1) we can find a convincing contribution of an absorption line. A weak absorption line with equivalent width down to $E W>$ $10 \mathrm{eV}$ can be excluded only in the (0.84-1.17) $\mathrm{keV}$ band, whereas a stronger line with $E W>200 \mathrm{eV}$ can be ruled out in the $(0.28-7.5) \mathrm{keV}$ band. According to the relation

$B \simeq 1.59 \times 10^{14} z_{\mathrm{G}}^{-1}\left(\frac{E_{\mathrm{c}}}{\mathrm{keV}}\right) \mathrm{G}$,

where $z_{\mathrm{G}}$ is the gravitational redshift, this corresponds to magnetic fields in the range of $(1.3-1.9) \times 10^{14} \mathrm{G}$ and $(0.44-12) \times$ $10^{14} \mathrm{G}$, respectively, for $z_{\mathrm{G}}=1$. However, we note that also no convincing detection of a proton cyclotron-absorption feature has been reported for magnetars up to now (Mereghetti 2008).

The long term $I$-band light curve, as measured with OGLEIV has been examined by Schmidtke et al. (2012a) and exhibits short term variability of $\sim 9$ days, that is likely an alias of sinusoidal modulations of $(0.9007 \pm 0.0005) \mathrm{d}$, caused by non-radial pulsations. In addition, two strong outbursts are seen reaching maximum emission around MJD $55500(\Delta I \sim 0.6 \mathrm{mag})$ and 
between MJD 56 151-56173 ( $\Delta I \gtrsim 0.15 \mathrm{mag})$. Schmidtke et al. (2012b) suggested the time-difference between the outbursts to be the likely orbital period $P_{\mathrm{o}}=(656 \pm 2) \mathrm{d}$. This is supported by the FRED-like shape (Bird et al. 2012) of the optical outbursts. If so, the X-ray emission in 2010 cannot be caused by a type-I X-ray outburst that can occur during periastron passage of the NS only.

This might be explained by a persistent X-ray state that is also favoured by the fact, that SXP 1062 was always detected above a flux of $\sim 10^{-12} \mathrm{erg} \mathrm{cm}^{-2} \mathrm{~s}^{-1}$ since its discovery. The pulsar must have entered this state after the last ROSAT observation. Comparing the deepest ROSAT upper limit of $F_{X} \leq$ $1.4 \times 10^{-13} \mathrm{erg} \mathrm{cm}^{-2} \mathrm{~s}^{-1}$ with the average flux of the 2010 $X M M-N e w t o n$ observations reveals an X-ray variability by a factor of 10 and by comparing with the brightest Swift detection, one obtains a maximum X-ray variability by a factor of at least 60 . The X-ray flux is also moderately (some 10\%) variable on short time scales (days), as measured with Swift and previously with Chandra (cf. Fig. 2 of Oskinova et al. 2013a).

The X-ray outburst around MJD 56200 may correlate with the second optical outburst. Because our Swift observations did not catch the initial stage of X-ray outburst we cannot judge about the outburst duration and maximum emission. The first Swift observation was performed 58 days after the first measured $I$-band increase. Regarding the long orbital period, this time interval might still be consistent with a type-I X-ray outburst. In this scenario, the NS accretes matter during periastron passage, leading to X-ray emission, while tidal forces affect the circumstellar disc. A sudden but local perturbation in the disc geometry, such as e.g. warping a part of the disc or the formation of a quasi-steady accretion disc around the NS, might cause the sharp increase in total optical luminosity. Crude estimates show that the observed increase in $I$-band magnitude can be achieved if the projected disc area increased by about $20 \%$.

The optical counterpart of SXP 1062, the B0III e star 2dFS 3831, was already observed spectroscopically by Evans et al. (2004) in Sep. 1998 (blue) and Sep. 1999 (red), the later $\sim 4$ months after the ASCA observation. On 2010 Oct. 25, 2dFS 3831 was observed spectroscopically with the VLT FLAMES instrument, only 4 days before the first optical outburst. These spectra are discussed in Hénault-Brunet et al. (2012). Our reported SALT spectrum was taken during the decline of the second outburst (dashed lines in Fig. 1).

The post-outburst SALT spectrum (see Fig. 5) is similar to those previously published and show that the Be star disc was at large not affected during the X-ray outburst, as it can be the case in type-II outbursts. The hydrogen Balmer lines have contributing emission filling them in, most likely as a result of a large circumstellar disc. This is also corroborated by a non-split shape of the $\mathrm{H} \alpha$ line, indicating the disc large in size and/or its inclination is small (Hummel \& Vrancken 1995). However, the $\mathrm{H} \beta$ and $\mathrm{H} \gamma$ emission lines show split shapes.

Despite overall similarity, there are some differences in spectra obtained at different epochs. The most apparent is the increasing strength of the $\mathrm{H} \gamma$ line occurring between the $2 \mathrm{dF}$ and the VLT measurement. In the SALT spectrum, the $\mathrm{H} \gamma$ emission line is still seen. The VLT spectrum shows a stronger red-ward wing contribution, while it is more symmetrical in the SALT spectrum pointing to V/R variability (see Fig. 6, right).

Another evidence for an increasing size or density of the disc is given by the increasing equivalent width of the $\mathrm{H} \alpha$ and $\mathrm{H} \beta$ emission lines between the $2 \mathrm{dF}$ and the SALT measurement by $(21.0 \pm 0.5) \%$ and $(52 \pm 21) \%$, respectively. Thus, the spectroscopic measurements suggest that the decretion disc has been growing over the last $\sim 14$ years. This may help to explain the non-detections of SXP 1062 by earlier X-ray missions (Sect. 3.3), because only a small or no decretion disc might have existed in the past (at least before 1999).

The optical and X-ray spectra of SXP 1062 are similar to those observed from other BeXRBs. The unusually slow spin period and its evolution (so far no spin-up was observed), hence, are even more surprising. The young long-period pulsar SXP 1062 continues to challenge the present models of accreting pulsars.

\section{Conclusions}

We presented the analysis of a recent XMM-Newton observation of SXP 1062 and discuss the results in the context of complementary Swift/XRT and optical data. The main results and conclusions are as follows:

1. The NS continued to spin down to a spin period of $P_{\mathrm{s}}=$ $(1071.01 \pm 0.16) \mathrm{s}$ as measured on 2012 Oct. 14. This implies an average $\dot{P}_{\mathrm{s}}=(2.27 \pm 0.44) \mathrm{s} \mathrm{yr}^{-1}$ during a baseline of 915 days.

2. We do not see significant spectral changes in the X-ray spectrum, compared to the 2010 observations, despite of an indication of a soft excess and $\mathrm{Fe} \mathrm{K}_{\alpha}$ at higher luminosities $\left(L_{\mathrm{X}}=3 \times 10^{36} \mathrm{erg} \mathrm{s}^{-1}\right)$.

3. We see indications of emission lines in the RGS spectra from prominent thermal emission lines. The SNR can unlikely account completely for this component.

4. No convincing indication of proton cyclotron-absorption lines are found. If we demand an absorption line with $E W>$ $200 \mathrm{eV}$, this would exclude a magnetic field of the NS between $4.4 \times 10^{13} \mathrm{G}$ and $1.2 \times 10^{15} \mathrm{G}$.

5. The X-ray light curve suggests that SXP 1062 is currently in a persistent $\mathrm{X}$-ray emitting state with a luminosity around $L_{\mathrm{X}}=8 \times 10^{35} \mathrm{erg} \mathrm{s}^{-1}$. Optical and X-ray outbursts might correlate and be caused by type-I outbursts.

6. The optical spectra obtained during the X-ray outburst are morphologically similar to the pre-outburst spectra obtained in 1998/99 and 2010. Strong Balmer emission lines, originating from the circumstellar disc of the Be star, are present during all observations. This indicate that the disc is large and not strongly affected by the X-ray outburst.

7. The evolution of the Balmer emission lines provides evidence for an increase of the circumstellar disc over the last $\sim 14$ years. This can explain the observed increase in the persistent X-ray luminosity of SXP 1062 during this time.

Acknowledgements. We thank the XMM-Newton team for scheduling the ToO observation. The XMM-Newton project is supported by the Bundesministerium für Wirtschaft und Technologie/Deutsches Zentrum für Luft- und Raumfahrt (BMWI/DLR, FKZ 50 OX 0001) and the Max-Planck Society. We acknowledge the use of public data from the Swift data archive and thank the Swift team for accepting and scheduling the ToO observation. Some of the observations reported in this paper were obtained with the Southern African Large Telescope (SALT). The OGLE project has received funding from the European Research Council under the European Community's Seventh Framework Programme (FP7/2007-2013)/ERC grant agreement No. 246678 to AU. MPES is funded through the Claude Leon Foundation Postdoctoral Fellowship program and the National Research Foundation. L.M.O. and RS acknowledge support from the BMWI/DLR grants FKZ 50 OR 1302 and FKZ 50 OR 0907, respectively. LMO acknowledges fruitful discussions within the international team at ISSI (International Space Science Institute) in Bern, especially with M.J. Torrjon and P. Kretschmar. J.S.G. thanks the University of Wisconsin-Madison College of Letters \& Science for partial support of this research and the campus for funding the Wisconsin participation in SALT. 


\section{References}

Arnaud, K. A. 1996, in Astronomical Data Analysis Software and Systems V, eds. G. H. Jacoby, \& J. Barnes, ASP Conf. Ser., 101, 17

Bildsten, L., Chakrabarty, D., Chiu, J., et al. 1997, ApJS, 113, 367

Bird, A. J., Coe, M. J., McBride, V. A., \& Udalski, A. 2012, MNRAS, 423, 3663

Buckley, D. A. H., Burgh, E. B., Cottrell, P. L., et al. 2006a, in SPIE Conf. Ser., $6269,62690 \mathrm{~A}$

Buckley, D. A. H., Swart, G. P., \& Meiring, J. G. 2006b, in SPIE Conf. Ser., $6267,62670 \mathrm{~A}$

Burgh, E. B., Nordsieck, K. H., Kobulnicky, H. A., et al. 2003, in SPIE Conf. Ser. 4841, eds. M. Iye, \& A. F. M. Moorwood, 1463

Cash, W. 1979, ApJ, 228, 939

Crawford, S. M., Still, M., Schellart, P., et al. 2010, in SPIE Conf. Ser., 7737, 12

den Herder, J. W., Brinkman, A. C., Kahn, S. M., et al. 2001, A\&A, 365, L7

Evans, C. J., Howarth, I. D., Irwin, M. J., Burnley, A. W., \& Harries, T. J. 2004, MNRAS, 353, 601

Fu, L., \& Li, X.-D. 2012, ApJ, 757, 171

Gregory, P. C., \& Loredo, T. J. 1996, ApJ, 473, 1059

Haberl, F., \& Pietsch, W. 2005, A\&A, 438, 211

Haberl, F., Filipović, M. D., Pietsch, W., \& Kahabka, P. 2000, A\&AS, 142, 41

Haberl, F., Eger, P., \& Pietsch, W. 2008, A\&A, 489, 327

Haberl, F., Sturm, R., Filipović, M. D., Pietsch, W., \& Crawford, E. J. 2012, A\&A, 537, L1

Hénault-Brunet, V., Oskinova, L. M., Guerrero, M. A., et al. 2012, MNRAS, 420, L13

Hickox, R. C., Narayan, R., \& Kallman, T. R. 2004, ApJ, 614, 881

Hummel, W., \& Vrancken, M. 1995, A\&A, 302, 751

Ikhsanov, N. R. 2012, MNRAS, 424, L39

Jansen, F., Lumb, D., Altieri, B., et al. 2001, A\&A, 365, L1
Kobulnicky, H. A., Nordsieck, K. H., Burgh, E. B., et al. 2003, in SPIE Conf Ser. 4841, eds. M. Iye, \& A. F. M. Moorwood, 1634

Mereghetti, S. 2008, A\&ARv, 15, 225

O’Donoghue, D., Buckley, D. A. H., Balona, L. A., et al. 2006, MNRAS, 372, 151

Oskinova, L. M., Guerrero, M. A., Hénault-Brunet, V., et al. 2013a, in IAU Symp., 291, 459

Oskinova, L. M., Sun, W., Evans, C. J., et al. 2013b, ApJ, 765, 73

Popov, S. B., \& Turolla, R. 2012, MNRAS, 421, L127

Pottschmidt, K., Suchy, S., Rivers, E., et al. 2012, in AIP Conf. Ser. 1427, eds. R. Petre, K. Mitsuda, \& L. Angelini, 60

Protassov, R., van Dyk, D. A., Connors, A., Kashyap, V. L., \& Siemiginowska, A. 2002, ApJ, 571, 545

Reig, P. 2011, Ap\&SS, 332, 1

Saxton, R. D., Read, A. M., Esquej, P., et al. 2008, A\&A, 480, 611

Schmidtke, P. C., Cowley, A. P., \& Udalski, A. 2012a, The Astronomer's Telegram, 4399, 1

Schmidtke, P. C., Cowley, A. P., \& Udalski, A. 2012b, The Astronomer's Telegram, 4596, 1

Strüder, L., Briel, U., Dennerl, K., et al. 2001, A\&A, 365, L18

Tody, D. 1993, in Astronomical Data Analysis Software and Systems II, eds. R. J. Hanisch, R. J. V. Brissenden, \& J. Barnes, ASP Conf. Ser., 52, 173

Turner, M. J. L., Abbey, A., Arnaud, M., et al. 2001, A\&A, 365, L27

Udalski, A. 2008, Acta Astron., 58, 187

Udalski, A., Szymanski, M. K., Soszynski, I., \& Poleski, R. 2008, Acta Astron., 58,69

Wang, Q., \& Wu, X. 1992, ApJS, 78, 391

Wilms, J., Allen, A., \& McCray, R. 2000, ApJ, 542, 914

Yokogawa, J., Imanishi, K., Tsujimoto, M., Koyama, K., \& Nishiuchi, M. 2003, PASJ, 55, 161

Zane, S., Turolla, R., Stella, L., \& Treves, A. 2001, ApJ, 560, 384 